\title{
On the Existence of Solutions for Impulsive Duffing Dynamic Equations on Time Scales with Dirichlet Boundary Conditions
}

\author{
Yongkun Li and Tianwei Zhang \\ Department of Mathematics, Yunnan University, Yunnan, Kunming 650091, China \\ Correspondence should be addressed to Yongkun Li, yklie@ynu.edu.cn \\ Received 4 June 2010; Accepted 16 August 2010 \\ Academic Editor: Douglas Robert Anderson
}

Copyright (C) 2010 Y. Li and T. Zhang. This is an open access article distributed under the Creative Commons Attribution License, which permits unrestricted use, distribution, and reproduction in any medium, provided the original work is properly cited.

By using critical point theory, some new sufficient conditions for the existence of solutions of impulsive Duffing dynamic equations on time scales with Dirichlet boundary conditions are obtained. Some examples are also given to illustrate our results.

\section{Introduction}

Consider the following Duffing dynamic equations on time scales with impulsive effects

$$
\begin{gathered}
u^{\Delta \Delta}(t)+C u^{\Delta}(\sigma(t))-r(t) u(\sigma(t))+f(\sigma(t), u(\sigma(t)))=h(t), \quad \text { a.e. } t \in[0, \sigma(T)]_{\mathbb{T}}^{\kappa^{2}}, \\
\Delta u^{\Delta}\left(t_{j}\right)=u^{\Delta}\left(t_{j}^{+}\right)-u^{\Delta}\left(t_{j}^{-}\right)=I_{j}\left(u\left(t_{j}\right)\right), \quad j=1,2, \ldots, p, \\
u(0)=0=u(\sigma(T)),
\end{gathered}
$$

where $T>0, C$ is a regressive constant, $t_{0}=0<t_{1}<t_{2}<\cdots<t_{p}<t_{p+1}=\sigma(T), r \in$ $L^{\infty}[0, \sigma(T)]_{\mathbb{T}}, h \in L^{2}[0, \sigma(T)]_{\mathbb{T}}, f:[0, \sigma(\sigma(T))]_{\mathbb{T}} \times \mathbb{R} \rightarrow \mathbb{R}$ is continuous, and $I_{j}: \times \mathbb{R} \rightarrow \mathbb{R}$, $j=1,2, \ldots, p$ are continuous.

Obviously, system (1.1) covers Duffing equations (when $\mathbb{T}=\mathbb{R}$ )

$$
\begin{gathered}
u^{\prime \prime}(t)+g(u(t))=h(t), \\
u^{\prime \prime}(t)+C u^{\prime}(t)+g(t, u(t))=h(t) .
\end{gathered}
$$


The Duffing equation has been used to model the nonlinear dynamics of special types of mechanical and electrical systems. This differential equation has been named after the studies of Duffing in 1918 [1], has a cubic nonlinearity, and describes an oscillator. The main applications have been in electronics, but it can also have applications in mechanics and in biology. For example, the brain is full of oscillators at micro- and macrolevel [2]. There are applications in neurology, ecology, secure communications, chaotic synchronization, and so on. Due to the rich behaviour of these equations, the most general forced forms of the Duffing equation (1.2) have been studied by many researchers [3-12].

The study of dynamic equations on time scales goes back to its founder Stefan Hilger [13], and is a new area of still fairly theoretical exploration in mathematics. Motivating the subject is the notion that dynamic equations on time scales can build bridges between continuous and discrete equations. Further, the study of time scales has led to several important applications, for example, in the study of insect population models, neural networks, heat transfer, and epidemic models [14-16].

Impulsive effects exist widely in many evolution processes in which their states are changed abruptly at certain moments of time. The theory of impulsive differential systems has been developed by numerous mathematicians (see [17-24]). Applications of impulsive differential equations with or without delays occur in biology, medicine, mechanics, engineering, chaos theory, and so on (see [25-32]).

In addition, system (1.1) also includes

$$
\begin{gathered}
u^{\Delta \Delta}(t)+f(\sigma(t), u(\sigma(t)))=0, \quad \text { a. e. } t \in[0, \sigma(T)]_{\mathbb{T}}^{\kappa^{2}}, \\
u(0)=0=u(\sigma(T)), \\
u^{\prime \prime}(t)+r(t) u(t)=f(t, u(t)), \quad \text { a.e. } t \in[0, T], \\
\Delta u^{\prime}\left(t_{j}\right)=u^{\prime}\left(t_{j}^{+}\right)-u^{\prime}\left(t_{j}^{-}\right)=I_{j}\left(t_{j}\right), \quad j=1,2, \ldots, p, \\
u(0)=0=u(T),
\end{gathered}
$$

which were studied by papers $[33,34]$, and some existence results were obtained by using some critical point theorems.

Our purpose in this paper is to study the variational structure of problem (1.1) in an appropriate space of functions and the existence of solutions for problem (1.1) by means of some critical point theorems. The organization of this paper is as follows. In Section 2, we make some preparations. In Section 3, we will study the variational structure of problem (1.1) and give some important lemmas which will be used in later section. In Section 4, by applying some critical point theorems, we establish sufficient conditions for the existence of solutions to problem (1.1). Some illustrative examples are also given in Section 4.

\section{Preliminaries}

In this section, we will first recall some basic definitions and lemmas which are used in what follows. 
Definition 2.1 (see [13]). A time scale $\mathbb{T}$ is an arbitrary nonempty closed subset of the real set $\mathbb{R}$ with the topology and ordering inherited from $\mathbb{R}$. The forward and backward jump operators $\sigma, \rho: \mathbb{T} \rightarrow \mathbb{T}$, and the graininess $\mu, v: \mathbb{T} \rightarrow \mathbb{R}^{+}$are defined, respectively, by

$$
\begin{aligned}
\sigma(t):=\inf \{s \in \mathbb{T}: s>t\}, & \rho(t):=\sup \{s \in \mathbb{T}: s<t\}, \\
\mu(t):=\sigma(t)-t, & \mathcal{v}(t):=t-\rho(t) .
\end{aligned}
$$

The point $t \in \mathbb{T}$ is called left dense, left scattered, right dense, or right scattered if $\rho(t)=t$, $\rho(t)<t, \sigma(t)=t$, or $\sigma(t)>t$, respectively. Points that are right dense and left dense at the same time are called dense. If $\mathbb{T}$ has a left-scattered maximum $m_{1}$, defined $\mathbb{T}^{\kappa}=\mathbb{T}-\left\{m_{1}\right\}$; otherwise, set $\mathbb{T}^{\mathcal{K}}=\mathbb{T}$. If $\mathbb{T}$ has a right-scattered minimum $m_{2}$, defined $\mathbb{T}_{\kappa}=\mathbb{T}-\left\{m_{2}\right\}$; otherwise, set $\mathbb{T}_{\kappa}=\mathbb{T}$.

Definition 2.2 (see [13]). For $f: \mathbb{T} \rightarrow \mathbb{R}$ and $t \in \mathbb{T}^{\kappa}$, then the delta derivative of $f$ at the point $t$ is defined to be the number $f^{\Delta}(t)$ (provided it exists) with the property that for each $\epsilon>0$, there is a neighborhood $U$ of $t$ such that

$$
\left|f(\sigma(t))-f(s)-f^{\Delta}(t)(\sigma(t)-s)\right| \leq \epsilon|\sigma(t)-s|, \quad \forall s \in U
$$

Definition 2.3 (see [13]). A function $f$ is rd continuous provided it is continuous at each right-dense point in $\mathbb{T}$ and has a left-sided limit at each left-dense point in $\mathbb{T}$. The set of rdcontinuous functions $f$ will be denoted by $C_{r d}(\mathbb{T})$.

Definition 2.4 (see [13]). Assume that $\mathbb{T}$ is an arbitrary time scale. We say that a function $p: \mathbb{T} \rightarrow \mathbb{R}$ is regressive provided

$$
1+\mu(t) p(t) \neq 0, \quad \forall t \in \mathbb{T}^{\kappa}
$$

Definition 2.5 (see [13]). Assume that $\mathbb{T}$ is an arbitrary time scale and a function $p: \mathbb{T} \rightarrow \mathbb{R}$ is regressive, then we define the exponential function on $\mathbb{T}$ by

$$
e_{p}(t, s)=\exp \left(\int_{s}^{t} \xi_{\mu(\tau)}(p(\tau)) \Delta \tau\right), \quad \text { for } s, t \in \mathbb{T}
$$

in which

$$
\xi_{h}(z)=\frac{1}{h} \log (1+z h) \quad \text { for } h>0,
$$

$\xi_{h}(z)=\xi_{0}(z)=z$ for $h=0$, here Log is the principal logarithm function.

A function $f: \mathbb{T} \rightarrow \mathbb{R}$ is called rd continuous provided it is continuous at each rightdense point and its left-sided limit exists (finite) at each left-dense point in $\mathbb{T}$. We write $f \in C_{r d}(\mathbb{T})=C_{r d}(\mathbb{T}, \mathbb{R})$. If $f$ is differentiable with $f^{\Delta} \in C_{r d}\left(\mathbb{T}^{\kappa}\right)$, we write $f \in C_{r d}^{1}(\mathbb{T})$. If $f^{\Delta}$ is differentiable on $\mathbb{T}^{\kappa^{2}}=\left(\mathbb{T}^{\kappa}\right)^{\kappa}$ with $f^{\Delta^{2}}=\left(f^{\Delta}\right)^{\Delta} \in C_{r d}\left(\mathbb{T}^{\kappa^{2}}\right)$, we write $f \in C_{r d}^{2}(\mathbb{T})$. 
Similar to the classical Riemann's definition of integrability, the concept of the Riemann delta integral on time scales is given in [35]. We know that many familiar functions, including monotone continuous, piecewise continuous, right-dense continuous functions, are Riemann delta integrable. In the following lemma, we present some properties of the integral that will be needed later.

Lemma 2.6 (see [35]). Let $f, g: \mathbb{T} \rightarrow \mathbb{R}$ be two functions and $a, b \in \mathbb{T}$. Then one has the following.

(1) Let $f$ and $g$ be Riemann delta integrable functions on $[a, b]$, and $\alpha, \beta \in \mathbb{R}$. Then $\alpha f+\beta g$ is Riemann delta integrable and

$$
\int_{a}^{b}(\alpha f(s)+\beta g(s)) \Delta s=\alpha \int_{a}^{b} f(s) \Delta s+\beta \int_{a}^{b} g(s) \Delta s .
$$

(2) Fundamental Theorem of Calculus. Let $f$ be a continuous function on $[a, b]_{\mathbb{T}}$ such that $f$ is delta differentiable on $[a, b)$. If $f^{\Delta}$ is Riemann delta integrable from $a$ to $b$, then

$$
\int_{a}^{b} f^{\Delta}(s) \Delta s=f(b)-f(a)
$$

(3) Integration by Parts. Let $f$ and $g$ be continuous functions on $[a, b]$ that are differentiable on $[a, b)$. If $f^{\Delta}$ and $g^{\Delta}$ are Riemann delta integrable from $a$ to $b$, then

$$
\int_{a}^{b} f(s) g^{\Delta}(s) \Delta s=\left.[f(s) g(s)]\right|_{a} ^{b}-\int_{a}^{b} f^{\Delta}(s) g(\sigma(s)) \Delta s .
$$

(4) If $f$ is Riemann delta integrable on $[a, b]$, then so is $|f|$, and

$$
\left|\int_{a}^{b} f^{\Delta}(s) \Delta s\right|=\int_{a}^{b}\left|f^{\Delta}(s)\right| \Delta s
$$
[35].

The construction of the $\Delta$-measure on $\mathbb{T}$ and the following concepts can be found in

(1) For each $t_{0} \in \mathbb{T} \backslash\{\max \mathbb{T}\}$, the single-point set $t_{0}$ is $\Delta$-measurable, and its $\Delta$-measure is given by

$$
\mu_{\Delta}\left(\left\{t_{0}\right\}\right)=\sigma\left(t_{0}\right)-t_{0}=\mu\left(t_{0}\right) .
$$


(2) If $a, b \in \mathbb{T}$ and $a \leq b$, then

$$
\mu_{\Delta}([a, b))=b-a, \quad \mu_{\Delta}((a, b))=b-\sigma(a) .
$$

(3) If $a, b \in \mathbb{T} \backslash\{\max \mathbb{T}\}$ and $a \leq b$, then

$$
\mu_{\Delta}((a, b])=\sigma(b)-\sigma(a), \quad \mu_{\Delta}([a, b])=\sigma(b)-a .
$$

The Lebesgue integral associated with the measure $\mu_{\Delta}$ on $\mathbb{T}$ is called the Lebesgue delta integral. For a (measurable) set $E \subset \mathbb{T}$ and a function $f: E \rightarrow \mathbb{R}$, the corresponding integral of $f$ on $E$ is denoted by $\int_{E} f(s) \Delta s$. All theorems of the general Lebesgue integration theory hold also for the Lebesgue delta integral on $\mathbb{T}$. Moreover, comparing the Lebesgue delta integral with the Riemann delta integral, we have the following.

Lemma 2.7 (see [35]). Let $[a, b]$ be a closed bounded interval in $\mathbb{T}$ and let $f$ be a bounded realvalued function defined on $[a, b]$. If $f$ is Riemann delta integrable from $a$ to $b$, then $f$ is Lebesgue delta integrable on $[a, b)$ and

$$
(R) \int_{a}^{b} f(s) \Delta s=(L) \int_{[a, b)} f(s) \Delta s
$$

where $R$ and $L$ indicate the Riemann delta integral and Lebesgue delta integral, respectively.

Assume that $p \in \mathbb{R}$ and $p>1$. Let $L_{L S}^{p}[a, b]$ be the set of

$$
\left\{f:[a, b] \longrightarrow \mathbb{R}: \int_{a}^{b}|f(s)|^{p} \Delta s<+\infty\right\}
$$

By Lemma 3.6 in $[36], L_{L S}^{p}[a, b]$ is a complete linear space with the norm $\|\cdot\|_{p}$ defined by

$$
\|f\|_{p}=\left(\int_{a}^{b}|f(s)|^{p} \Delta s\right)^{1 / p}
$$

Let $C_{T S}[a, b]$ denote the linear space of all continuous functions $f:[a, b] \rightarrow \mathbb{R}$ with the maximum norm $\|f\|_{C}=\max _{t \in[a, b]}|f(t)|$.

Lemma 2.8 (Hölder inequality [37]). Let $f, g \in C_{r d}([a, b]), p>1$ and $q$ the conjugate number of p. Then

$$
\int_{a}^{b}|f(s) g(s)| \Delta s \leq\left(\int_{a}^{b}|f(s)|^{p} \Delta s\right)^{1 / p} \cdot\left(\int_{a}^{b}|g(s)|^{q} \Delta s\right)^{1 / q} .
$$


At the end of this section, we recall some notation and known results from critical point theory.

Let $\mathbb{X}$ be a real normed space and let $\varphi$ be a functional from $\mathbb{X}$ to $\mathbb{R}$.

(1) $\varphi$ is called weakly continuous if

$$
x_{k} \rightarrow x \Longrightarrow \varphi\left(x_{k}\right) \longrightarrow \varphi(x) .
$$

(2) $\varphi$ is called lower semicontinuous if

$$
x_{k} \longrightarrow x \Longrightarrow \lim _{k \rightarrow \infty} \inf \varphi\left(x_{k}\right) \geq \varphi(x) .
$$

(3) $\varphi$ is called weakly lower semicontinuous if

$$
x_{k} \rightarrow x \Longrightarrow \lim _{k \rightarrow \infty} \inf \varphi\left(x_{k}\right) \geq \varphi(x) .
$$

Let $\mathbb{X}$ be a real Hilbert space, $\varphi \in C^{1}(\mathbb{X}, \mathbb{R})$, which means that $\varphi$ is a continuously Fréchet-differentiable functional defined on $\mathbb{X} . \varphi$ is said to satisfy the Palais-Smale condition (P.S. condition) if any sequence $\left\{x_{n}\right\} \subset \mathbb{X}$ for which $\left\{\varphi\left(x_{n}\right)\right\}$ is bounded and $\varphi^{\prime}\left(x_{n}\right) \rightarrow 0$ as $n \rightarrow \infty$ possesses a convergent subsequence in $\mathbb{X}$.

Let $B_{r}$ be the open ball in $\mathbb{X}$ with radius $r$ and centered at 0 and let $\partial B_{r}$ denote its boundary. The following lemma is taken from $[38,39]$ and will play an important role in the proof of our main results.

Lemma 2.9 (see [38]). If $\mathbb{X}$ is a real normed space and $\varphi: \mathbb{X} \rightarrow \mathbb{R}$ is lower semicontinuous and convex, then $\varphi$ is weakly lower semicontinuous.

Lemma 2.10 (see [39]). Let $\mathbb{X}$ be a real Banach space, $\varphi \in C^{1}(\mathbb{X}, \mathbb{R})$ be weakly lower(upper) semicontinuous, and

$$
\lim _{\|x\| \rightarrow \infty} \varphi(x)=+\infty \quad\left(\lim _{\|x\| \rightarrow \infty} \varphi(x)=-\infty\right) .
$$

Then, there exists $x_{0} \in \mathbb{X}$ such that

$$
\varphi\left(x_{0}\right)=\inf _{x \in \mathbb{X}} \varphi(x) \quad\left(\varphi\left(x_{0}\right)=\sup _{x \in \mathbb{X}} \varphi(x)\right) .
$$

Lemma 2.11 (Mountain Pass Theorem [40]). Let $\mathbb{X}$ be a real Hilbert space and let $\varphi \in C^{1}(\mathbb{X}, \mathbb{R})$ satisfy P.S. condition, and $\varphi$ satisfies

(J1) There are constants $\rho, \alpha>0$ such that $\varphi(x) \geq \alpha$ for all $x \in \partial B_{\rho}$, where $B_{\rho}=\{x \in \mathbb{X}$ : $\|x\|<\rho\}$.

(J2) $\varphi(0) \leq 0$ and there exists $x_{0} \bar{\in} B_{\rho}$ such that $\varphi\left(x_{0}\right) \leq 0$. 
Then $\varphi$ possesses a critical value $c \geq \alpha$. Moreover, $c$ can be characterized as

$$
c=\inf _{g \in \Gamma} \max _{s \in[0,1]} \varphi(g(s))
$$

where

$$
\Gamma=\left\{g \in C([0,1], \mathbb{X}) \mid g(0)=0, g(1)=x_{0}\right\}
$$

\section{Variational Structure}

In this section, we will establish the corresponding variational framework for problem (1.1). Let $\mathbb{P}$ be the function space of the form

$$
\mathbb{P}=\left\{u \mid u \in C_{r d}^{1}[0, \sigma(T)]_{\mathbb{T}}^{\kappa}, u \in C_{T S}[0, \sigma(T)]_{\mathbb{T}}, u(0)=0=u(\sigma(T))\right\}
$$

$\mathbb{P}$ can be equipped with the inner product

$$
(u, v)_{\mathbb{P}}=\int_{0}^{\sigma(T)} u^{\Delta}(t) v^{\Delta}(t) \Delta t, \quad \forall u, v \in \mathbb{P}
$$

inducing the norm

$$
\|u\|_{\mathbb{P}}=\sqrt{(u, u)_{\mathbb{P}}}=\left(\int_{0}^{\sigma(T)}\left|u^{\Delta}(t)\right|^{2} \Delta t\right)^{1 / 2}, \quad \forall u \in \mathbb{P} .
$$

Let $\left\{u_{k}\right\} \in \mathbb{P}$ be a Cauchy sequence in $\mathbb{P}$, that is, $\left\|u_{k}^{\Delta}-u_{l}^{\Delta}\right\|_{2}=\left\|u_{k}-u_{l}\right\|_{\mathbb{P}} \rightarrow 0$ as $k, l \rightarrow \infty$. Since $L_{L S}^{2}[0, \sigma(T)]_{\mathbb{T}}$ is a complete space, there exists a $v \in L_{L S}^{2}[0, \sigma(T)]_{\mathbb{T}}$ such that $\left\|u_{k}^{\Delta}-v\right\|_{2} \rightarrow 0$ as $k \rightarrow \infty$. Define a function $u$ by

$$
u(t)=\int_{0}^{t} v(s) \Delta s
$$

The function $v$ is called a weak derivative of $u$ and is also denoted by $u^{\Delta}$.

Remark 3.1. If $u$ has a weak derivative, then this weak derivative is unique in $L_{L S}^{2}[0, \sigma(T)]_{\mathbb{T}}$, that is, if $v_{1}, v_{2} \in L_{L S}^{2}[0, \sigma(T)]_{\mathbb{T}}$ are both weak derivatives of $u$, then $\left\|v_{1}-v_{2}\right\|_{2}=0$. If $u$ is (delta) differential on $[0, \sigma(T)]_{\mathbb{T}}$, then its weak derivative is its (delta) derivative. 
From (3.4), it is clear that $u(0)=0$ and

$$
\begin{aligned}
|u(\sigma(T))| & =\left|\int_{0}^{\sigma(T)} v(s) \Delta s\right| \\
& =\left|\int_{0}^{\sigma(T)}\left(v(s)-u_{k}^{\Delta}(s)\right) \Delta s\right| \\
& \leq \int_{0}^{\sigma(T)}\left|v(s)-u_{k}^{\Delta}(s)\right| \Delta s \\
& \leq\left(\int_{0}^{\sigma(T)} 1^{2} \Delta s\right)^{1 / 2}\left(\int_{0}^{\sigma(T)}\left|v(s)-u_{k}^{\Delta}(s)\right|^{2} \Delta s\right)^{1 / 2} \\
& =\sqrt{\sigma(T)}\left\|v-u_{k}^{\Delta}\right\|_{2} \longrightarrow 0, \text { as } k \longrightarrow \infty
\end{aligned}
$$

Thus, $u(\sigma(T))=0$.

Define the space $\mathbb{H}$ as

$$
\mathbb{H}=\left\{u(t)=\int_{0}^{t} v(s) \Delta s \mid \exists\left\{u_{k}\right\} \in \mathbb{P} \text { satisfying }\left\|u_{k}^{\Delta}-v\right\|_{2} \longrightarrow 0 \text { as } k \longrightarrow \infty\right\} .
$$

It is clear that $\mathbb{P} \subset \mathbb{H}$, and we define the inner product $(\cdot, \cdot)_{\mathbb{H}}$ in $\mathbb{H}$ as

$$
(u, v)_{\mathbb{H}}=\int_{0}^{\sigma(T)} u^{\Delta}(t) v^{\Delta}(t) \Delta t, \quad \forall u, v \in \mathbb{H},
$$

inducing the norm

$$
\|u\|_{\mathbb{H}}=\sqrt{(u, u)_{\mathbb{H}}}=\left(\int_{0}^{\sigma(T)}\left|u^{\Delta}(t)\right|^{2} \Delta t\right)^{1 / 2}, \quad \forall u \in \mathbb{H},
$$

where $u^{\Delta}$ represents the weak derivative of $u$. Moreover, we have the following result.

Lemma 3.2 (see [33]). $\mathbb{H}$ is a complete space in the norm $\|\cdot\|_{\mathbb{H}}$ and inequality

$$
\int_{0}^{\sigma(T)}\left|u^{\sigma}(t)\right|^{2} \Delta t \leq \sigma(T)^{2} \int_{0}^{\sigma(T)}\left|u^{\Delta}(t)\right|^{2} \Delta t, \quad \forall u \in \mathbb{H}
$$

holds, that is, $\left\|u^{\sigma}\right\|_{2} \leq \sigma(T)\|u\|_{\mathbb{H}}$.

Also, we consider the inner product

$$
(u, v)=\int_{0}^{\sigma(T)} e_{C}(t, 0) r(t) u^{\sigma}(t) v^{\sigma}(t) \Delta t+\int_{0}^{\sigma(T)} e_{C}(t, 0) u^{\Delta}(t) v^{\Delta}(t) \Delta t, \quad \forall u, v \in \mathbb{H},
$$


inducing the norm

$$
\|u\|=\left(\int_{0}^{\sigma(T)} e_{C}(t, 0) r(t)\left|u^{\sigma}(t)\right|^{2} \Delta t+\int_{0}^{\sigma(T)} e_{C}(t, 0)\left|u^{\Delta}(t)\right|^{2} \Delta t\right)^{1 / 2}, \quad \forall u \in \mathbb{H} .
$$

Throughout this paper, it will be assumed that ess $\inf _{t \in[0, \sigma(T)]_{T}} r(t)=r_{0}>-\lambda_{0}$, where $\lambda_{0}$ is the smallest eigenvalue of the problem

$$
\begin{gathered}
u^{\Delta \Delta}(t)+\lambda u^{\sigma}(t)=0, \quad t \in[0, \sigma(T)]_{\mathbb{T}}^{\kappa^{2}} \\
u(0)=0=u(\sigma(T)) .
\end{gathered}
$$

From [41], $\lambda_{0}$ may be defined as

$$
\lambda_{0}=\min _{\substack{u \neq 0 \\ u(0)=0=u(\sigma(T))}}-\frac{\int_{0}^{\sigma(T)} u^{\Delta \Delta}(t) u^{\sigma}(t) \Delta t}{\left\|u^{\sigma}\right\|_{2}^{2}} .
$$

Applying the integration by parts in Lemma 2.6, we have

$$
\lambda_{0}=\min _{\substack{u \neq 0 \\ u(0)=0=u(\sigma(T))}} \frac{\|u\|_{\mathbb{H}}^{2}}{\left\|u^{\sigma}\right\|_{2}^{2}}>0,
$$

which implies that

$$
\left\|u^{\sigma}\right\|_{2}^{2} \leq \frac{1}{\lambda_{0}}\|u\|_{\mathbb{H}}^{2}, \quad \forall u \in \mathbb{H} .
$$

For convenience, we introduce the following notations:

$$
e^{l}=\min _{t \in[0, \sigma(T)]_{\mathbb{T}}} e_{C}(t, 0), \quad e^{L}=\max _{t \in[0, \sigma(T)]_{\mathbb{T}}} e_{C}(t, 0), \quad h_{0}=\int_{0}^{\sigma(T)}|h(t)| \Delta t .
$$

Lemma 3.3. Suppose that

(H1) $r_{0} e^{L}+\lambda_{0} e^{l}>0$, for all $r_{0} \in\left(-\lambda_{0}, 0\right)$.

Then one has

$$
\omega\|u\|_{\mathbb{H}}^{2} \leq\|u\|^{2} \leq e^{L}\left(\|r\|_{\infty} \sigma^{2}(T)+1\right)\|u\|_{\mathbb{H}}^{2},
$$

which implies that the norm $\|\cdot\|$ and the norm $\|\cdot\|_{\mathbb{H}}$ are equivalent, where

$$
\omega= \begin{cases}\frac{r_{0} e^{L}}{\lambda_{0}}+e^{l}, & -\lambda_{0}<r_{0}<0 \\ e^{l}, & r_{0} \geq 0\end{cases}
$$


Proof

Case $1\left(r_{0} \geq 0\right)$. For every $u \in \mathbb{H}$, we have

$$
\begin{aligned}
\|u\|^{2} & =\int_{0}^{\sigma(T)} e_{C}(t, 0) r(t)\left|u^{\sigma}(t)\right|^{2} \Delta t+\int_{0}^{\sigma(T)} e_{C}(t, 0)\left|u^{\Delta}(t)\right|^{2} \Delta t \\
& \geq \int_{0}^{\sigma(T)} e_{C}(t, 0)\left|u^{\Delta}(t)\right|^{2} \Delta t \\
& \geq e^{l} \int_{0}^{\sigma(T)}\left|u^{\Delta}(t)\right|^{2} \Delta t \\
& =e^{l}\|u\|_{\mathbb{H}}^{2} \cdot
\end{aligned}
$$

On the other hand, by Lemma 2.6, one has

$$
\begin{aligned}
\|u\|^{2} & =\int_{0}^{\sigma(T)} e_{C}(t, 0) r(t)\left|u^{\sigma}(t)\right|^{2} \Delta t+\int_{0}^{\sigma(T)} e_{C}(t, 0)\left|u^{\Delta}(t)\right|^{2} \Delta t \\
& \leq e^{L}\left(\int_{0}^{\sigma(T)}\left|r(t) \| u^{\sigma}(t)\right|^{2} \Delta t+\int_{0}^{\sigma(T)}\left|u^{\Delta}(t)\right|^{2} \Delta t\right) \\
& \leq e^{L}\left(\|r\|_{\infty} \sigma^{2}(T)+1\right) \int_{0}^{\sigma(T)}\left|u^{\Delta}(t)\right|^{2} \Delta t \\
& =e^{L}\left(\|r\|_{\infty} \sigma^{2}(T)+1\right)\|u\|_{\mathbb{H}}^{2} .
\end{aligned}
$$

Therefore, the norm $\|\cdot\|$ and the norm $\|\cdot\|_{\mathbb{H}}$ are equivalent.

Case $2\left(-\lambda_{0}<r_{0}<0\right)$. For every $u \in \mathbb{H}$, we have from (3.15) that

$$
\begin{aligned}
\|u\|^{2} & =\int_{0}^{\sigma(T)} e_{C}(t, 0) r(t)\left|u^{\sigma}(t)\right|^{2} \Delta t+\int_{0}^{\sigma(T)} e_{C}(t, 0)\left|u^{\Delta}(t)\right|^{2} \Delta t \\
& \geq e^{L} r_{0} \int_{0}^{\sigma(T)}\left|u^{\sigma}(t)\right|^{2} \Delta t+e^{l} \int_{0}^{\sigma(T)}\left|u^{\Delta}(t)\right|^{2} \Delta t \\
& \geq \frac{r_{0} e^{L}}{\lambda_{0}} \int_{0}^{\sigma(T)}\left|u^{\Delta}(t)\right|^{2} \Delta t+e^{l} \int_{0}^{\sigma(T)}\left|u^{\Delta}(t)\right|^{2} \Delta t \\
& =\left(\frac{r_{0} e^{L}}{\lambda_{0}}+e^{l}\right) \int_{0}^{\sigma(T)}\left|u^{\Delta}(t)\right|^{2} \Delta t \\
& =\left(\frac{r_{0} e^{L}}{\lambda_{0}}+e^{l}\right)\|u\|_{\mathbb{H}}^{2} \cdot
\end{aligned}
$$

Similar to Case 1, it is easy to obtain the desired results. This completes the proof. 
Together with (3.15) and Lemma 3.3, it is not difficult to obtain the following.

Lemma 3.4 (Poincaré inequality). For all $u \in \mathbb{H}$, one has

$$
\int_{0}^{\sigma(T)}\left|u^{\sigma}(t)\right|^{2} \Delta t \leq \frac{1}{\lambda_{0} \omega}\left(\int_{0}^{\sigma(T)} e_{C}(t, 0) r(t)\left|u^{\sigma}(t)\right|^{2} \Delta t+\int_{0}^{\sigma(T)} e_{C}(t, 0)\left|u^{\Delta}(t)\right|^{2} \Delta t\right)
$$

that is, $\left\|u^{\sigma}\right\|_{2} \leq\left(1 / \sqrt{\lambda_{0} \omega}\right)\|u\|$.

Lemma 3.5. $\|u\|_{\infty} \leq a_{0}\|u\|$ for all $u \in \mathbb{H}$, where $a_{0}=\sqrt{\sigma(T) / \omega}$.

Proof. For every $u \in \mathbb{H}$, we have

$$
\begin{aligned}
|u(t)| & =|u(t)-u(0)| \\
& =\left|\int_{0}^{t} u^{\Delta}(s) \Delta s\right| \\
& \leq \int_{0}^{t}\left|u^{\Delta}(s)\right| \Delta s \\
& \leq t^{1 / 2}\left(\int_{0}^{t}\left|u^{\Delta}(s)\right|^{2} \Delta s\right)^{1 / 2} \\
& \leq t^{1 / 2}\|u\|_{\mathbb{H}} .
\end{aligned}
$$

Therefore,

$$
\|u\|_{\infty} \leq \sqrt{\sigma(T)}\|u\|_{\mathbb{H}} \leq \sqrt{\frac{\sigma(T)}{\omega}}\|u\|
$$

This completes the proof.

For $u \in \mathbb{H} \cap C_{r d}^{2}[0, \sigma(T)]_{\mathbb{T}}^{\kappa^{2}}$, we have that $u$ and $u^{\Delta}$ are both absolutely continuous. Hence, $\Delta u^{\Delta}(t)=u^{\Delta}\left(t^{+}\right)-u^{\Delta}\left(t^{-}\right)=0$ for any $t \in[0, \sigma(T)]_{\mathbb{T}}^{\kappa}$.

If $u \in \mathbb{H}$, then $u$ is absolutely continuous and $u^{\Delta} \in L_{T S}^{2}[0, \sigma(T)]_{\mathbb{T}}$. In this case, $\Delta u^{\Delta}(t)=$ $u^{\Delta}\left(t^{+}\right)-u^{\Delta}\left(t^{-}\right)=0$ may not hold for some $t \in[0, \sigma(T)]_{\mathbb{T}}^{\kappa}$. It leads to the impulsive effects.

Take $v \in \mathbb{H}$ and multiply the two sides of the equality

$$
u^{\Delta \Delta}(t)+C u^{\Delta}(\sigma(t))-r(t) u(\sigma(t))+f(\sigma(t), u(\sigma(t)))=h(t)
$$


by $e_{C}(t, 0), v^{\sigma}(t)$ and integrate from 0 to $\sigma(T)$; we have

$$
\begin{aligned}
\int_{0}^{\sigma(T)}\left(e_{C}(t, 0) u^{\Delta}(t)\right)^{\Delta} v^{\sigma}(t) \Delta t= & \int_{0}^{\sigma(T)} e_{C}(t, 0) h(t) v^{\sigma}(t) \Delta t \\
& -\int_{0}^{\sigma(T)} e_{C}(t, 0) r(t) u(\sigma(t)) v^{\sigma}(t) \Delta t \\
& -\int_{0}^{\sigma(T)} e_{C}(t, 0) f(\sigma(t), u(\sigma(t))) v^{\sigma}(t) \Delta t .
\end{aligned}
$$

Furthermore,

$$
\begin{aligned}
\int_{0}^{\sigma(T)} & \left(e_{C}(t, 0) u^{\Delta}(t)\right)^{\Delta} v^{\sigma}(t) \Delta t \\
= & \sum_{j=0}^{j=p} \int_{t_{j}}^{t_{j+1}}\left(e_{C}(t, 0) u^{\Delta}(t)\right)^{\Delta} v^{\sigma}(t) \Delta t \\
= & \sum_{j=0}^{j=p}\left(e_{C}\left(t_{j+1}^{-}, 0\right) u^{\Delta}\left(t_{j+1}^{-}\right) v\left(t_{j+1}^{-}\right)-e_{C}\left(t_{j}^{+}, 0\right) u^{\Delta}\left(t_{j}^{+}\right) v\left(t_{j}^{+}\right)-\int_{t_{j}}^{t_{j+1}} e_{C}(t, 0) u^{\Delta}(t) v^{\Delta}(t) \Delta t\right) \\
= & -\sum_{j=1}^{j=p} e_{C}\left(t_{j}, 0\right) \Delta u^{\Delta}\left(t_{j}\right) v\left(t_{j}\right)+e_{C}(\sigma(T), 0) u^{\Delta}(\sigma(T)) v(\sigma(T))-e_{C}(0,0) u^{\Delta}(0) v(0) \\
& -\int_{0}^{\sigma(T)} e_{C}(t, 0) u^{\Delta}(t) v^{\Delta}(t) \Delta t \\
= & -\sum_{j=1}^{j=p} e_{C}\left(t_{j}, 0\right) I_{j}\left(u\left(t_{j}\right)\right) v\left(t_{j}\right)-\int_{0}^{\sigma(T)} e_{C}(t, 0) u^{\Delta}(t) v^{\Delta}(t) \Delta t .
\end{aligned}
$$

Combining (3.26), we have

$$
\begin{gathered}
\int_{0}^{\sigma(T)} e_{C}(t, 0) u^{\Delta}(t) v^{\Delta}(t) \Delta t+\int_{0}^{\sigma(T)} e_{C}(t, 0) r(t) u(\sigma(t)) v^{\sigma}(t) \Delta t+\sum_{j=1}^{j=p} e_{C}\left(t_{j}, 0\right) I_{j}\left(u\left(t_{j}\right)\right) v\left(t_{j}\right) \\
=\int_{0}^{\sigma(T)} e_{C}(t, 0) f(\sigma(t), u(\sigma(t))) v^{\sigma}(t) \Delta t-\int_{0}^{\sigma(T)} e_{C}(t, 0) h(t) v^{\sigma}(t) \Delta t .
\end{gathered}
$$

Considering the above, we introduce the following concept solution for problem (1.1). 
Definition 3.6. We say that a function $u \in \mathbb{H}$ is a weak solution of problem (1.1) if the identity

$$
\begin{gathered}
\int_{0}^{\sigma(T)} e_{C}(t, 0) u^{\Delta}(t) v^{\Delta}(t) \Delta t+\int_{0}^{\sigma(T)} e_{C}(t, 0) r(t) u(\sigma(t)) v^{\sigma}(t) \Delta t+\sum_{j=1}^{p} e_{C}\left(t_{j}, 0\right) I_{j}\left(u\left(t_{j}\right)\right) v\left(t_{j}\right) \\
=\int_{0}^{\sigma(T)} e_{C}(t, 0) f(\sigma(t), u(\sigma(t))) v^{\sigma}(t) \Delta t-\int_{0}^{\sigma(T)} e_{C}(t, 0) h(t) v^{\sigma}(t) \Delta t
\end{gathered}
$$

holds for any $v \in \mathbb{H}$.

Consider the functional $\varphi: \mathbb{H} \rightarrow \mathbb{R}$ defined by

$$
\varphi(u)=\frac{1}{2}\|u\|^{2}+\int_{0}^{\sigma(T)} e_{C}(t, 0)\left[h(t) u^{\sigma}(t)-F\left(\sigma(t), u^{\sigma}(t)\right)\right] \Delta t+\sum_{j=1}^{p} e_{C}\left(t_{j}, 0\right) \int_{0}^{u\left(t_{j}\right)} I_{j}(s) \mathrm{d} s,
$$

where $F(t, u)=\int_{0}^{u} f(t, s) \mathrm{d} s$. Using the continuity of $f$ and $I_{j}, j=1,2, \ldots, p$, one has that $\varphi \in C^{1}(\mathbb{H}, \mathbb{R})$. For any $v \in \mathbb{H}$, we have

$$
\begin{aligned}
\varphi^{\prime}(u) v= & \int_{0}^{\sigma(T)} e_{C}(t, 0) u^{\Delta}(t) v^{\Delta}(t) \Delta t+\int_{0}^{\sigma(T)} e_{C}(t, 0) r(t) u^{\sigma}(t) v^{\sigma}(t) \Delta t \\
& -\int_{0}^{\sigma(T)} e_{C}(t, 0) f\left(\sigma(t), u^{\sigma}(t)\right) v^{\sigma}(t) \Delta t+\int_{0}^{\sigma(T)} e_{C}(t, 0) h(t) v^{\sigma}(t) \Delta t \\
& +\sum_{j=1}^{p} e_{C}\left(t_{j}, 0\right) I_{j}\left(u\left(t_{j}\right)\right) v\left(t_{j}\right) .
\end{aligned}
$$

Therefore, the solutions of problem (1.1) are the corresponding critical points of $\varphi$.

Lemma 3.7 (see [33]). The functionals $\psi_{1}, \psi_{2}, \psi_{3}: \mathbb{H} \rightarrow \mathbb{R}$ defined by

$$
\psi_{1}(u)=\int_{0}^{\sigma(T)} F\left(\sigma(t), u^{\sigma}(t)\right) \Delta t, \quad \psi_{2}(u)=\int_{0}^{\sigma(T)} h(t) u^{\sigma}(t) \Delta t, \quad \psi_{3}(u)=\sum_{j=1}^{p} \int_{0}^{u\left(t_{j}\right)} I_{j}(s) \mathrm{d} s
$$

are weakly continuous on $\mathbb{H}$. 


\section{Main Results}

Theorem 4.1. Assume that (H1) holds. Suppose further the following.

(H2) There exist $a, b>0$ and $\theta \in[0,1)$ such that

$$
|f(t, u)| \leq a+b|u|^{\theta}, \quad \forall(t, u) \in[0, \sigma(T)]_{\mathbb{T}} \times \mathbb{R} .
$$

(H3) There exist $a_{j}, b_{j}>0$ and $\theta_{j} \in[0,1)(j=1,2, \ldots, p)$ such that

$$
\left|I_{j}(u)\right| \leq a_{j}+b_{j}|u|^{\theta_{j}}, \quad \forall u \in \mathbb{R}(j=1,2, \ldots, p) .
$$

Then problem (1.1) has at least one weak solution.

Proof. According to Lemma 3.7, $\psi_{1}(u), \psi_{2}(u), \psi_{3}(u)$ are weakly continuous on $\mathbb{H}$. On the other hand, it is clear that $\|u\|^{2}$ is continuous and convex on $\mathbb{H}$. By Lemma $2.9,\|u\|^{2}$ is weakly lower semicontinuous. So $\varphi(u)$ is weakly lower semicontinuous on $\mathbb{H}$.

From $(\mathrm{H} 2)-(\mathrm{H} 3)$, we have

$$
\begin{aligned}
\varphi(u)= & \frac{1}{2}\|u\|^{2}+\int_{0}^{\sigma(T)} e_{C}(t, 0)\left[h(t) u^{\sigma}(t)-F\left(\sigma(t), u^{\sigma}(t)\right)\right] \Delta t+\sum_{j=1}^{p} e_{C}\left(t_{j}, 0\right) \int_{0}^{u\left(t_{j}\right)} I_{j}(s) \mathrm{d} s \\
\geq & \frac{1}{2}\|u\|^{2}-e^{L} \int_{0}^{\sigma(T)}\left|h(t) u^{\sigma}(t)\right| \Delta t-e^{L} \int_{0}^{\sigma(T)}\left(a\left|u^{\sigma}(t)\right|+b\left|u^{\sigma}(t)\right|^{\theta+1}\right) \Delta t \\
& -e^{L} \sum_{j=1}^{p} \int_{0}^{u\left(t_{j}\right)}\left(a_{j}+b_{j}|s|^{\theta_{j}}\right) \mathrm{d} s \\
\geq & \frac{1}{2}\|u\|^{2}-e^{L} h_{0}\|u\|_{\infty}-e^{L} \sigma(T)\left(a\|u\|_{\infty}+b\|u\|_{\infty}^{\theta+1}\right)-e^{L} \sum_{j=1}^{p}\left(a_{j}\|u\|_{\infty}+b_{j}\|u\|_{\infty}^{\theta_{j}+1}\right) \\
\geq & \frac{1}{2}\|u\|^{2}-e^{L} h_{0} a_{0}\|u\|-e^{L} \sigma(T)\left(a a_{0}\|u\|+b a_{0}^{\theta+1}\|u\|^{\theta+1}\right) \\
& -e^{L} \sum_{j=1}^{p}\left(a_{j} a_{0}\|u\|+b_{j} a_{0}^{\theta_{j}+1}\|u\|^{\theta_{j}+1}\right)
\end{aligned}
$$


for all $u \in \mathbb{H}$. This implies that $\lim _{\|u\| \rightarrow \infty} \varphi(u)=\infty$, and $\varphi$ is coercive. By Lemma 2.10, $\varphi$ has a minimum point on $\mathbb{H}$, which is a critical point of $\varphi$. Therefore, problem (1.1) has at least one weak solution. This completes the proof.

Example 4.2. Consider the following impulsive Duffing equations on time scales

$$
\begin{gathered}
u^{\Delta \Delta}(t)+2 u^{\Delta}(t)-3 u(t)+\sqrt{|u(t)|}=\frac{1}{10 e} \sin t, \quad \text { a.e. } t \in[0,1]_{\mathbb{T}}, \\
\Delta u^{\Delta}\left(\frac{1}{2}\right)=u^{\Delta}\left(\frac{1}{2}^{+}\right)-u^{\Delta}\left(\frac{1^{-}}{2}\right)=\sqrt[3]{u\left(\frac{1}{2}\right)} \\
u(0)=0=u(\sigma(1)) .
\end{gathered}
$$

Then, problem (4.4) has at least one solution.

Proof. The result is easy to obtain from Theorem 4.1 and we should omit it. This completes the proof.

Theorem 4.3. Assume that (H1) holds. Suppose further the following.

(H4) There exist $a, b, c>0$ and $\theta \in[0,1)$ such that

$$
|f(t, u)| \leq a|u|+b|u|^{\theta}+c, \quad \forall(t, u) \in[0, \sigma(T)]_{\mathbb{T}} \times \mathbb{R}
$$

(H5) There exist $a_{j}, b_{j}, c_{j}>0$ and $\theta_{j} \in[0,1)(j=1,2, \ldots, p)$ such that

$$
\left|I_{j}(u)\right| \leq a_{j}|u|+b_{j}|u|^{\theta_{j}}+c_{j}, \quad \forall u \in \mathbb{R}(j=1,2, \ldots, p) .
$$

(H6) $1 / 2-a \sigma(T) e^{L} a_{0}^{2}-\sum_{j=1}^{p} a_{j} e^{L} a_{0}^{2}>0$.

Then problem (1.1) has at least one weak solution.

Proof. According to Theorem 4.1, we know that $\varphi(u)$ is weakly lower semicontinuous on $\mathbb{H}$. From (H4)-(H6), we have

$$
\begin{aligned}
\varphi(u)= & \frac{1}{2}\|u\|^{2}+\int_{0}^{\sigma(T)} e_{C}(t, 0)\left[h(t) u^{\sigma}(t)-F\left(\sigma(t), u^{\sigma}(t)\right)\right] \Delta t+\sum_{j=1}^{p} e_{C}\left(t_{j}, 0\right) \int_{0}^{u\left(t_{j}\right)} I_{j}(s) \mathrm{d} s \\
\geq & \frac{1}{2}\|u\|^{2}-e^{L} \int_{0}^{\sigma(T)}\left|h(t) u^{\sigma}(t)\right| \Delta t-e^{L} \int_{0}^{\sigma(T)}\left(a\left|u^{\sigma}(t)\right|^{2}+b\left|u^{\sigma}(t)\right|^{\theta+1}+c\left|u^{\sigma}(t)\right|\right) \Delta t \\
& -e^{L} \sum_{j=1}^{p} \int_{0}^{u\left(t_{j}\right)}\left(a_{j}|s|+b_{j}|s|^{\theta_{j}}+c_{j}\right) \mathrm{d} s
\end{aligned}
$$




$$
\begin{aligned}
\geq & \frac{1}{2}\|u\|^{2}-e^{L} h_{0}\|u\|_{\infty}-e^{L} \sigma(T)\left(a\|u\|_{\infty}^{2}+b\|u\|_{\infty}^{\theta+1}+c\|u\|_{\infty}\right) \\
& -e^{L} \sum_{j=1}^{p}\left(a_{j}\|u\|_{\infty}^{2}+b_{j}\|u\|_{\infty}^{\theta_{j}+1}+c_{j}\|u\|_{\infty}\right) \\
\geq & \frac{1}{2}\|u\|^{2}-e^{L} h_{0} a_{0}\|u\|-e^{L} \sigma(T)\left(a a_{0}^{2}\|u\|^{2}+b a_{0}^{\theta+1}\|u\|^{\theta+1}+c a_{0}\|u\|\right) \\
& -e^{L} \sum_{j=1}^{p}\left(a_{j} a_{0}^{2}\|u\|^{2}+b_{j} a_{0}^{\theta_{j}+1}\|u\|^{\theta_{j}+1}+c_{j} a_{0}\|u\|\right) \\
= & \left(\frac{1}{2}-a \sigma(T) e^{L} a_{0}^{2}-\sum_{j=1}^{p} a_{j} e^{L} a_{0}^{2}\right)\|u\|^{2}-e^{L} h_{0} a_{0}\|u\| \\
& -e^{L} \sigma(T)\left(b a_{0}^{\theta+1}\|u\|^{\theta+1}+c a_{0}\|u\|\right)-e^{L} \sum_{j=1}^{p}\left(b_{j} a_{0}^{\theta_{j}+1}\|u\|^{\theta_{j}+1}+c_{j} a_{0}\|u\|\right)
\end{aligned}
$$

for all $u \in \mathbb{H}$. This implies that $\lim _{\|u\| \rightarrow \infty} \varphi(u)=\infty$, and $\varphi$ is coercive. By Lemma 2.10, $\varphi$ has a minimum point on $\mathbb{H}$, which is a critical point of $\varphi$. Therefore, problem (1.1) has at least one weak solution. This completes the proof.

Example 4.4. Consider the following impulsive Duffing equations on time scales:

$$
\begin{gathered}
u^{\Delta \Delta}(t)+2 u^{\Delta}(t)-3 u(t)+\frac{1}{5 \sigma^{3}(1) v_{0}} u(t)+\sqrt{|u(t)|}=\sin t, \quad \text { a.e. } t \in[0,1]_{\mathbb{T}}, \\
\Delta u^{\Delta}\left(\frac{1}{2}\right)=u^{\Delta}\left(\frac{1^{+}}{2}\right)-u^{\Delta}\left(\frac{1^{-}}{2}\right)=\frac{1}{5 \sigma^{2}(1) v_{0}} u\left(\frac{1}{2}\right), \\
u(0)=0=u(\sigma(1)),
\end{gathered}
$$

where

$$
v_{0}=\exp \left(\int_{0}^{\sigma(1)} \log (1+3 \mu(s))^{1 / \mu(s)} \Delta s\right)
$$

Then problem (4.8) has at least one solution.

Proof. By an easy calculation, we obtain

$$
e^{L}=\exp \left(\int_{0}^{\sigma(1)} \log (1+3 \mu(s))^{1 / \mu(s)} \Delta s\right), \quad e^{l}=1
$$

It is easy to verify that all the conditions of Theorem 4.3 are satisfied. From Theorem 4.3, problem (4.8) has at least one solution. This completes the proof. 
Lemma 4.5. Assume that (H1) and (H3) hold. Suppose further the following.

(H7) There exist constants $\mu>2$ and $R>0$, such that

$$
0<\mu F(t, u) \leq u f(t, u), \quad \forall|u| \geq R, t \in[0, \sigma(T)]_{\mathbb{T}},
$$

where $F(t, u)=\int_{0}^{u} f(t, s) \mathrm{d} s$.

Then $\varphi$ satisfies the P.S. condition in $\mathbb{H}$.

Proof. Let $\left\{u_{n}\right\}$ be a sequence in $\mathbb{H}$ satisfying that $\left\{\varphi\left(u_{n}\right)\right\}$ is bounded and $\varphi^{\prime}\left(u_{n}\right) \rightarrow 0$ as $n \rightarrow+\infty$. Let $\Omega_{n}=\left\{t \in[0, \sigma(T)]_{\mathbb{T}}|| u_{n}(t) \mid \geq R\right\}$ for all $n \in \mathbb{N}$. By the continuity of $f$ and (H7), there exists a constant $c_{1}>0$ such that

$$
\begin{aligned}
\beta \varphi\left(u_{n}\right)-\left(\varphi^{\prime}\left(u_{n}\right), u_{n}\right)= & \left(\frac{\beta}{2}-1\right)\left\|u_{n}\right\|^{2}+\int_{0}^{\sigma(T)} e_{C}(t, 0)\left[u_{n}^{\sigma} f\left(\sigma(t), u_{n}^{\sigma}\right)-\beta F\left(\sigma(t), u_{n}^{\sigma}\right)\right] \Delta t \\
& +\beta \sum_{j=1}^{p} e_{C}\left(t_{j}, 0\right) \int_{0}^{u_{n}\left(t_{j}\right)} I_{j}(s) \mathrm{d} s-\sum_{j=1}^{p} e_{C}\left(t_{j}, 0\right) I_{j}\left(u_{n}\left(t_{j}\right)\right) u_{n}\left(t_{j}\right) \\
& +\int_{0}^{\sigma(T)}(\beta-1) e_{C}(t, 0) h(t) u_{n}^{\sigma} \Delta t \\
\geq & \left(\frac{\beta}{2}-1\right)\left\|u_{n}\right\|^{2}+\int_{\Omega_{n}} e_{C}(t, 0)\left[u_{n}^{\sigma} f\left(\sigma(t), u_{n}^{\sigma}\right)-\beta F\left(\sigma(t), u_{n}^{\sigma}\right)\right] \Delta t \\
& -(\beta+1) e^{L} \sum_{j=1}^{p}\left(a_{j} a_{0}\|u\|+b_{j} a_{0}^{\theta_{j}+1}\|u\|^{\theta_{j}+1}\right) \\
& -(\beta-1) e^{L} h_{0} a_{0}\left\|u_{n}\right\|-c_{1} \\
\geq & \left(\frac{\beta}{2}-1\right)\left\|u_{n}\right\|^{2}-(\beta+1) e^{L} \sum_{j=1}^{p}\left(a_{j} a_{0}\|u\|+b_{j} a_{0}^{\theta_{j}+1}\|u\|^{\theta_{j}+1}\right) \\
& -(\beta-1) e^{L} h_{0} a_{0}\left\|u_{n}\right\|-c_{1},
\end{aligned}
$$

which implies that $\left\{u_{n}\right\}$ is bounded in $\mathbb{H}$. Taking into account $\left\{u_{n}\right\} \subset \mathbb{H} \subset C_{T S}[0, \sigma(T)]_{\mathbb{T}}$ and employing Lemma 3.5 , we know that $\left\{u_{n}\right\}$ is relatively compact in $C_{T S}[0, \sigma(T)]_{\mathbb{T}}$. Thus $\left\{u_{n}\right\}$ possesses a convergent subsequence $\left\{u_{n_{k}}\right\}$ in $C_{T S}[0, \sigma(T)]_{\mathbb{T}}$, that is, $\left\|u_{n_{i}}-u_{n_{j}}\right\|_{\infty} \rightarrow 0$ as $i, j \rightarrow+\infty$. since $u_{n} \in \mathbb{H}$, we know that $u_{n}^{\sigma}(T)=0$ for all $n \in \mathbb{N}$. From the above inequality, 
it is easy to see that

$$
\sup _{t \in[0, \sigma(T)]_{\mathbb{T}}^{\kappa}}\left|u_{n_{i}}(t)-u_{n_{j}}(t)\right| \longrightarrow 0, \quad \text { as } i, j \longrightarrow+\infty \text {. }
$$

From the continuity of $f$ and $I_{j}, j=1,2, \ldots, p$, we have

$$
\left|f\left(\sigma(t), u_{n_{i}}^{\sigma}(t)\right)-f\left(\sigma(t), u_{n_{j}}^{\sigma}(t)\right)\right| \longrightarrow 0, \quad\left|I_{j}\left(u_{n_{i}}(t)\right)-I_{j}\left(u_{n_{j}}(t)\right)\right| \longrightarrow 0
$$

hold uniformly for $t \in[0, \sigma(T)]_{\mathbb{T}}^{\kappa}$ as $i, j \rightarrow+\infty$. Set

$$
\begin{gathered}
\left\|f\left(\sigma(t), u_{n_{i}}^{\sigma}(t)\right)-f\left(\sigma(t), u_{n_{j}}^{\sigma}(t)\right)\right\|_{\infty}=\sup _{t \in[0, \sigma(T)]_{\mathbb{T}}^{\kappa}} \mid f\left(\sigma(t), u_{n_{i}}^{\sigma}(t)\right)-f\left(\sigma(t), u_{n_{j}}^{\sigma}(t)\right), \\
\left\|I_{j}\left(u_{n_{i}}(t)\right)-I_{j}\left(u_{n_{j}}(t)\right)\right\|_{\infty}=\sup _{t \in[0, \sigma(T)]_{\mathbb{T}}^{\kappa}}\left|I_{j}\left(u_{n_{i}}(t)\right)-I_{j}\left(u_{n_{j}}(t)\right)\right| .
\end{gathered}
$$

Clearly,

$$
\left\|f\left(\sigma(t), u_{n_{i}}^{\sigma}(t)\right)-f\left(\sigma(t), u_{n_{j}}^{\sigma}(t)\right)\right\|_{\infty} \longrightarrow 0, \quad\left\|I_{j}\left(u_{n_{i}}(t)\right)-I_{j}\left(u_{n_{j}}(t)\right)\right\|_{\infty} \longrightarrow 0
$$

as $i, j \rightarrow+\infty$. We rewrite (3.31) as

$$
\begin{aligned}
\left(\varphi^{\prime}(u), v\right)= & (u, v)-\int_{0}^{\sigma(T)} e_{C}(t, 0) f(\sigma(t), u(\sigma(t))) v^{\sigma}(t) \Delta t \\
& +\int_{0}^{\sigma(T)} e_{C}(t, 0) h(t) v^{\sigma}(t) \Delta t+\sum_{j=1}^{p} e_{C}\left(t_{j}, 0\right) I_{j}\left(u\left(t_{j}\right)\right) v\left(t_{j}\right)
\end{aligned}
$$

for any $u, v \in \mathbb{H}$. Therefore,

$$
\begin{aligned}
\left(u_{n_{i}}-u_{n_{j}}, v\right)= & \left(\varphi^{\prime}\left(u_{n_{i}}\right)-\varphi^{\prime}\left(u_{n_{j}}\right), v\right)-\sum_{j=1}^{p} e_{C}\left(t_{j}, 0\right)\left[I_{j}\left(u_{n_{i}}\left(t_{j}\right)\right)-I_{j}\left(u_{n_{j}}\left(t_{j}\right)\right)\right] v\left(t_{j}\right) \\
& +\int_{0}^{\sigma(T)} e_{C}(t, 0)\left[f\left(\sigma(t), u_{n_{i}}(\sigma(t))\right)-f\left(\sigma(t), u_{n_{j}}(\sigma(t))\right)\right] v^{\sigma}(t) \Delta t .
\end{aligned}
$$


Thus, for any $v \in \mathbb{H}$, it follows that

$$
\begin{aligned}
\left|\left(u_{n_{i}}-u_{n_{j}}, v\right)\right| \leq & \left\|\varphi^{\prime}\left(u_{n_{i}}\right)-\varphi^{\prime}\left(u_{n_{j}}\right)\right\|\|v\|+e^{L} \sum_{j=1}^{p}\left\|I_{j}\left(u_{n_{i}}\left(t_{j}\right)\right)-I_{j}\left(u_{n_{j}}\left(t_{j}\right)\right)\right\|_{\infty}\left\|v\left(t_{j}\right)\right\|_{\infty} \\
& +e^{L}\left\|f\left(\sigma(t), u_{n_{i}}(\sigma(t))\right)-f\left(\sigma(t), u_{n_{j}}(\sigma(t))\right)\right\|_{\infty} \int_{0}^{\sigma(T)}\left|v^{\sigma}(t)\right| \Delta t \\
\leq & \left\|\varphi^{\prime}\left(u_{n_{i}}\right)-\varphi^{\prime}\left(u_{n_{j}}\right)\right\|\|v\|+e^{L} a_{0} \sum_{j=1}^{p}\left\|I_{j}\left(u_{n_{i}}\left(t_{j}\right)\right)-I_{j}\left(u_{n_{j}}\left(t_{j}\right)\right)\right\|_{\infty}\|v\| \\
& +e^{L} \sqrt{\sigma(T)}\left\|f\left(\sigma(t), u_{n_{i}}(\sigma(t))\right)-f\left(\sigma(t), u_{n_{j}}(\sigma(t))\right)\right\|_{\infty}\left\|v^{\sigma}\right\|_{2} \\
\leq & \left\|\varphi^{\prime}\left(u_{n_{i}}\right)-\varphi^{\prime}\left(u_{n_{j}}\right)\right\|\|v\|+e^{L} a_{0} \sum_{j=1}^{p}\left\|I_{j}\left(u_{n_{i}}\left(t_{j}\right)\right)-I_{j}\left(u_{n_{j}}\left(t_{j}\right)\right)\right\|_{\infty}\|v\| \\
& +e^{L} \frac{\sqrt{\sigma(T)}}{\sqrt{\lambda_{0} \omega}}\left\|f\left(\sigma(t), u_{n_{i}}(\sigma(t))\right)-f\left(\sigma(t), u_{n_{j}}(\sigma(t))\right)\right\|_{\infty}\|v\| .
\end{aligned}
$$

Hence,

$$
\begin{aligned}
\left\|u_{n_{i}}-u_{n_{j}}\right\| & =\sup _{\|v\| \leq 1}\left|\left(u_{n_{i}}-u_{n_{j}}, v\right)\right| \\
\leq & \left\|\varphi^{\prime}\left(u_{n_{i}}\right)-\varphi^{\prime}\left(u_{n_{j}}\right)\right\|+e^{L} a_{0} \sum_{j=1}^{p}\left\|I_{j}\left(u_{n_{i}}\left(t_{j}\right)\right)-I_{j}\left(u_{n_{j}}\left(t_{j}\right)\right)\right\|_{\infty} \\
& +e^{L} \frac{\sqrt{\sigma(T)}}{\sqrt{\lambda_{0} \omega}}\left\|f\left(\sigma(t), u_{n_{i}}(\sigma(t))\right)-f\left(\sigma(t), u_{n_{j}}(\sigma(t))\right)\right\|_{\infty} .
\end{aligned}
$$

Observing that $\varphi^{\prime}\left(u_{n}\right) \rightarrow 0$ as $n \rightarrow+\infty$, it is easy to see that

$$
\left\|\varphi^{\prime}\left(u_{n_{i}}\right)-\varphi^{\prime}\left(u_{n_{j}}\right)\right\| \longrightarrow 0, \quad \text { as } i, j \longrightarrow+\infty
$$

Together with (4.16)-(4.21), we get

$$
\left\|u_{n_{i}}-u_{n_{j}}\right\| \longrightarrow 0, \quad \text { as } i, j \longrightarrow+\infty
$$

In other words, $\left\{u_{n}\right\}$ possesses a convergent subsequence $\left\{u_{n_{k}}\right\}$ in $\mathbb{H}$. The P.S. condition is now satisfied. This completes the proof. 
Theorem 4.6. Assume that (H1), (H3), and (H7) hold. Suppose further the following.

(H8) There exists constant $v \geq 2$ such that

$$
0<v F(t, u) \leq u f(t, u), \quad \forall u \in[-1,0) \bigcup(0,1], t \in[0, \sigma(T)]_{\mathbb{T}},
$$

(H9) $a_{0}^{2} / 2-e^{L} M \sigma(T)-e^{L} \sqrt{\sigma(T)}\|h\|_{2}-e^{L} \sum_{j=1}^{p} a_{j}-e^{L} \sum_{j=1}^{p} b_{j}>0$, where

$$
M:=\sup \left\{F(t, u): t \in[0, \sigma(T)]_{\mathbb{T}},|u|=1\right\} .
$$

Then problem (1.1) has at least one weak solution.

Proof. It suffices to show that $\varphi$ possesses a nonzero critical point. Now, we need to verify that all assumptions of the Mountain Pass Theorem hold. The P.S. condition follows from Lemma 4.5. Next, we will check conditions (J1) and (J2) of Lemma 2.11. Assume that $0<$ $\|u\|_{\infty} \leq 1$. In view of $(\mathrm{H} 8)$, for $\xi \in(0,1]$, we get that

$$
\frac{\mathrm{d}}{\mathrm{d} \xi}\left[F\left(t, \xi^{-1} u\right) \xi^{\nu}\right]=\frac{\mathrm{d}}{\mathrm{d} \xi}\left[\int_{0}^{\xi^{-1} u} f(t, s) \mathrm{d} s \xi^{\nu}\right]=\left(v F\left(t, \xi^{-1} u\right)-\xi^{-1} u f\left(t, \xi^{-1} u\right)\right) \xi^{\nu-1} \leq 0 .
$$

Hence,

$$
F(t, u) \leq F\left(t, \frac{u}{|u|}\right)|u|^{v}, \quad \text { for } 0<|u| \leq 1, t \in[0, \sigma(T)]_{\mathbb{T}},
$$

which implies that

$$
\begin{aligned}
\int_{0}^{\sigma(T)} e_{C}(t, 0)\left|F\left(\sigma(t), u^{\sigma}(t)\right)\right| \Delta t & \leq \int_{0}^{\sigma(T)} e_{C}(t, 0)\left|F\left(\sigma(t), \frac{u^{\sigma}(t)}{\left|u^{\sigma}(t)\right|}\right)\right|\left|u^{\sigma}(t)\right|^{v} \Delta t \\
& \leq e^{L} M \int_{0}^{\sigma(T)}\left|u^{\sigma}(t)\right|^{2} \Delta t \\
& \leq e^{L} M \frac{\sigma^{2}(T)}{\omega}\|u\|^{2} .
\end{aligned}
$$


At the same time, we can obtain that

$$
\begin{aligned}
\int_{0}^{\sigma(T)} e_{C}(t, 0)\left|h(t) u^{\sigma}(t)\right| \Delta t & \leq e^{L}\|h\|_{2}\left\|u^{\sigma}\right\|_{2} \leq e^{L} \frac{\sigma(T)}{\sqrt{\omega}}\|h\|_{2}\|u\|, \\
\sum_{j=1}^{p} e_{C}\left(t_{j}, 0\right) \int_{0}^{u\left(t_{j}\right)}\left|I_{j}(s)\right| \mathrm{d} s & \leq e^{L} \sum_{j=1}^{p} \int_{0}^{u\left(t_{j}\right)}\left(a_{j}+b_{j}|s|^{\theta_{j}}\right) \mathrm{d} s \\
& \leq e^{L} \sum_{j=1}^{p}\left(a_{j}\|u\|_{\infty}+b_{j}\|u\|_{\infty}^{\theta_{j}+1}\right) \\
& \leq e^{L} \sum_{j=1}^{p}\left(a_{j} a_{0}\|u\|+b_{j} a_{0}^{\theta_{j}+1}\|u\|^{\theta_{j}+1}\right) .
\end{aligned}
$$

Together (4.27)-(4.28) and the expression of functional $\varphi$, it follows that

$$
\begin{aligned}
\varphi(u) \geq & \frac{1}{2}\|u\|^{2}-e^{L} M \sigma(T) a_{0}^{2}\|u\|^{2}-e^{L} \sqrt{\omega} a_{0}^{2}\|h\|_{2}\|u\| \\
& -e^{L} \sum_{j=1}^{p}\left(a_{j} a_{0}\|u\|+b_{j} a_{0}^{\theta_{j}+1}\|u\|^{\theta_{j}+1}\right) \\
= & \left(\frac{1}{2}-e^{L} M \sigma(T) a_{0}^{2}\right)\|u\|^{2}-e^{L} \sum_{j=1}^{p} b_{j} a_{0}^{\theta_{j}+1}\|u\|_{j}^{\theta_{j}+1} \\
& -\left(e^{L} \sqrt{\omega} a_{0}^{2}\|h\|_{2}+e^{L} \sum_{j=1}^{p} a_{j} a_{0}\right)\|u\| \\
= & \left(\frac{1}{2}-e^{L} M \sigma(T) a_{0}^{2}-e^{L} \sqrt{\sigma(T)} a_{0}^{2}\|h\|_{2}-e^{L} \sum_{j=1}^{p} a_{j} a_{0}^{2}\right)\|u\|^{2} \\
& +\left(e^{L} \sqrt{\sigma(T)} a_{0}^{2}\|h\|_{2}+e^{L} \sum_{j=1}^{p} a_{j} a_{0}^{2}\right)\|u\|^{2} \\
& -\left(e^{L} \sqrt{\omega} a_{0}^{2}\|h\|_{2}+e^{L} \sum_{j=1}^{p} a_{j} a_{0}\right)\|u\| \\
& -e^{L} \sum_{j=1}^{p} b_{j} a_{0}^{\theta_{j}+1}\|u\|_{j}^{\theta_{j}+1} .
\end{aligned}
$$

Setting

$$
\|u\|=\rho:=\frac{1}{a_{0}}=\sqrt{\frac{\omega}{\sigma(T)}} .
$$


Then we have

$$
\left(e^{L} \sqrt{\sigma(T)} a_{0}^{2}\|h\|_{2}+e^{L} \sum_{j=1}^{p} a_{j} a_{0}^{2}\right) \rho^{2}-\left(e^{L} \sqrt{\omega} a_{0}^{2}\|h\|_{2}+e^{L} \sum_{j=1}^{p} a_{j} a_{0}\right) \rho=0,
$$

which implies from (H9) that

$$
\begin{aligned}
\varphi(u) & \geq\left(\frac{1}{2}-e^{L} M \sigma(T) a_{0}^{2}-e^{L} \sqrt{\sigma(T)} a_{0}^{2}\|h\|_{2}-e^{L} \sum_{j=1}^{p} a_{j} a_{0}^{2}\right) \rho^{2}-e^{L} \sum_{j=1}^{p} b_{j} a_{0}^{\theta_{j}+1} \rho^{\theta_{j}+1} \\
& =\frac{a_{0}^{2}}{2}-e^{L} M \sigma(T)-e^{L} \sqrt{\sigma(T)}\|h\|_{2}-e^{L} \sum_{j=1}^{p} a_{j}-e^{L} \sum_{j=1}^{p} b_{j} \\
& =\alpha>0 .
\end{aligned}
$$

By Lemma 3.5, if $\|u\|=\rho$, then $0<\|u\|_{\infty} \leq 1$. Set $B_{\rho}=\left\{u \in \mathbb{H} \mid\|u\|<\rho=1 / a_{0}\right\}$, then $\varphi(u) \geq \alpha>0$ for all $u \in \partial B_{\rho}$. Condition (J1) is satisfied. have

Secondly, we verify the condition (J2) of Lemma 2.11. In view of assumption (H7), we

$$
\begin{array}{cl}
\frac{\mu}{u} \leq \frac{f(t, u)}{F(t, u)}, \quad u \geq R \\
\frac{\mu}{u} \geq \frac{f(t, u)}{F(t, u)}, \quad u \leq-R .
\end{array}
$$

Integrating (4.33) and (4.34) for $u$ on $[R, u]$ and $[u,-R]$, respectively, we get

$$
\begin{gathered}
\mu \ln \frac{u}{R} \leq \ln \frac{F(t, u)}{F(t, u)}, \quad u \geq R, \\
\mu \ln \frac{R}{-u} \geq \ln \frac{F(t,-R)}{F(t, u)}, \quad u \leq-R,
\end{gathered}
$$

that is,

$$
\begin{gathered}
F(t, u) \geq F(t, R)\left(\frac{u}{R}\right)^{\mu}, \quad u \geq R, \\
F(t, u) \geq F(t,-R)\left(\frac{-u}{R}\right)^{\mu}, \quad u \leq-R .
\end{gathered}
$$

Combining (4.36) and (4.37), one has

$$
F(t, u) \geq \alpha_{1}|u|^{\mu}, \quad|u| \geq R
$$


where

$$
\alpha_{1}=R^{-\mu} \min \left\{\min _{t \in[0, \sigma(T)]_{\mathbb{T}}} F(t, R), \min _{t \in[0, \sigma(T)]_{\mathbb{T}}} F(t,-R)\right\}>0 .
$$

On the other hand, by the continuity of $F(t, u), F(t, u)$ is bounded on $[0, \sigma(T)]_{\mathbb{T}} \times[-R, R]$, there exists $K>0$ such that

$$
F(t, u) \geq-K \geq \alpha_{1}|u|^{\mu}-\alpha_{1} R^{\mu}-K, \quad|u| \leq R
$$

Combining (4.38) and (4.40), we have

$$
F(t, u) \geq \alpha_{1}|u|^{\mu}-\alpha_{2}, \quad \forall(t, u) \in[0, \sigma(T)]_{\mathbb{T}} \times \mathbb{R},
$$

where $\alpha_{2}=\alpha_{1} R^{\mu}+K$.

According to the Hölder inequality, we get

$$
\int_{0}^{\sigma(T)}\left|u^{\sigma}(t)\right|^{2} \Delta t \leq \sigma(T)^{1-2 / \mu}\left(\int_{0}^{\sigma(T)}\left|u^{\sigma}(t)\right|^{\mu} \Delta t\right)^{2 / \mu}
$$

Thus,

$$
\int_{0}^{\sigma(T)}\left|u^{\sigma}(t)\right|^{\mu} \Delta t \geq \sigma(T)^{(2-\mu) / 2}\left(\int_{0}^{\sigma(T)}\left|u^{\sigma}(t)\right|^{2} \Delta t\right)^{\mu / 2}=\sigma(T)^{(2-\mu) / 2}\left\|u^{\sigma}\right\|_{2}^{\mu} .
$$

Together (4.41) and (4.43), we know that

$$
\begin{aligned}
\int_{0}^{\sigma(T)} e_{C}(t, 0) F\left(\sigma(t), u^{\sigma}(t)\right) \Delta t & \geq \int_{0}^{\sigma(T)} e_{C}(t, 0) \alpha_{1}\left|u^{\sigma}(t)\right|^{\mu} \Delta t-\int_{0}^{\sigma(T)} \alpha_{2} e_{C}(t, 0) \Delta t \\
& \geq \alpha_{1} e^{l} \sigma(T)^{(2-\mu) / 2}\left\|u^{\sigma}\right\|_{2}^{\mu}-\alpha_{2} e^{L} \sigma(T) \\
& \geq \alpha_{3}\left\|u^{\sigma}\right\|_{2}^{\mu}-\alpha_{4}
\end{aligned}
$$

where $\alpha_{3}=\alpha_{1} e^{l} \sigma(T)^{(2-\mu) / 2}$, and $\alpha_{4}=\alpha_{2} e^{L} \sigma(T)$. 
Obviously, $\varphi(0)=0$. Let $v_{0} \in \mathbb{H}$ satisfy $\left\|v_{0}\right\|=1$. For any $\varsigma>0$, it follows from (4.44) and (4.28) that

$$
\begin{aligned}
\varphi\left(\varsigma v_{0}\right)= & \frac{\varsigma^{2}}{2}\left\|v_{0}\right\|^{2}-\int_{0}^{\sigma(T)} e_{C}(t, 0) F\left(\sigma(t), \varsigma v_{0}^{\sigma}(t)\right) \Delta t \\
& +\varsigma \int_{0}^{\sigma(T)} e_{C}(t, 0) h(t) v_{0}^{\sigma}(t) \Delta t+\sum_{j=1}^{p} e_{C}\left(t_{j}, 0\right) \int_{0}^{s_{0}\left(t_{j}\right)} I_{j}(s) \mathrm{d} s \\
\leq & \frac{\varsigma^{2}}{2}\left\|v_{0}\right\|^{2}-\alpha_{3} \varsigma^{\mu}\left\|v_{0}^{\sigma}\right\|_{2}^{\mu}+\varsigma e^{L} \frac{\sigma(T)}{\sqrt{\omega}}\|h\|_{2}\left\|v_{0}\right\| \\
& +e^{L} \sum_{j=1}^{p}\left(\varsigma a_{j} a_{0}\left\|v_{0}\right\|+b_{j} \varsigma^{\theta_{j}+1} a_{0}^{\theta_{j}+1}\left\|v_{0}\right\|^{\theta_{j}+1}\right)+\alpha_{4} .
\end{aligned}
$$

Since $\mu>2$, we see that $\varphi\left(\varsigma v_{0}\right) \rightarrow-\infty$ as $\varsigma \rightarrow+\infty$. we can choose sufficiently large $\varsigma_{0}$ such that $u_{0}=\varsigma_{0} v_{0} \in \mathbb{H}$ satisfying $u_{0} \bar{\in} \partial \bar{B}_{\rho}$ and $\varphi\left(u_{0}\right)<0$. Condition (J2) is satisfied.

According to Lemma 2.11, $\varphi$ possesses a critical value $\mathrm{c} \geq \alpha>0$ given by

$$
c=\inf _{g \in \Gamma} \max _{s \in[0,1]} \varphi(g(s)),
$$

where

$$
\Gamma=\left\{g \in C([0,1], \mathbb{E}) \mid g(0)=0, g(1)=u_{0}\right\} .
$$

Therefore, problem (1.1) has at least one weak solution. This completes the proof.

Example 4.7. Let $\mathbb{T}=\mathbb{R}$. Consider the following impulsive Duffing equations with Dirichlet boundary condition

$$
\begin{gathered}
u^{\prime \prime}(t)+\frac{1}{2} u^{\prime}(t)-u(t)+f(t, u(t))=\frac{1}{10 e} \sin t, \quad \text { a.e. } t \in[0,1] \\
\Delta u^{\prime}\left(\frac{1}{2}\right)=u^{\prime}\left(\frac{1^{+}}{2}\right)-u^{\prime}\left(\frac{1^{-}}{2}\right)=\frac{1}{10 e} \sqrt[5]{u\left(\frac{1}{2}\right)} \\
u(0)=0=u(1),
\end{gathered}
$$

where

$$
f(t, u)= \begin{cases}\frac{u}{10 e^{t+1}}, & \forall(t, u) \in([0,1] \times[-1,1]) \\ \frac{u^{3}}{10 e^{t+1}}, & \forall(t, u) \in([0,1] \times(-\infty,-1) \bigcup(1,+\infty)) .\end{cases}
$$

Then problem (4.48) has at least one solution. 
Proof. By an easy calculation, $e^{L}=e, e^{l}=1,\|h\|_{2} \leq 1 / 10 e, a_{0}=1, a_{1}=0, b_{1}=1 / 10 e$, and $M=1 / 20 e$. It is easy to verify that all the conditions of Theorem 4.6 are satisfied. From Theorem 4.6, problem (4.48) has at least one solution. This completes the proof.

Corollary 4.8. Assume that (H1), (H3), (H7), and (H8) hold. Suppose further the following.

(H10) $I_{j}(t) \geq 0$ for all $t \in[0, \sigma(T)]_{\mathbb{T}}, j=1,2, \ldots, p$.

(H11) $a_{0}^{2} / 2-e^{L} M \sigma(T)-e^{L} \sqrt{\sigma(T)}\|h\|_{2}>0$, where

$$
M:=\sup \left\{F(t, u): t \in[0, \sigma(T)]_{\mathbb{T}},|u|=1\right\} .
$$

Then problem (1.1) has at least one weak solution.

Corollary 4.9. Assume that (H1), (H3), (H7), (H8), and (H10) hold. Suppose further the following.

(H12) $h(t) \equiv 0$ for all $t \in[0, \sigma(T)]_{\mathbb{T}}$.

(H13) $a_{0}^{2} / 2>e^{L} M \sigma(T)$, where $M:=\sup \left\{F(t, u): t \in[0, \sigma(T)]_{\mathbb{T}},|u|=1\right\}$.

Then problem (1.1) has at least one weak solution.

Corollary 4.10. Assume that (H1), (H3), (H7), (H10), and (H12) hold. Suppose further the following.

$(\mathrm{H} 14) \sup _{t \in[0, \sigma(T)]_{\mathbb{T}}} \lim _{s \rightarrow 0}(f(t, s) / s)=0$.

Then problem (1.1) has at least one weak solution.

Proof. From the proof of Theorem 4.6, it is easy to see that $\varphi \in C^{1}(\mathbb{E}, \mathbb{R})$ and the condition (J2) of Lemma 2.11 is satisfied. We only need to verify the condition (J1) in Lemma 2.11.

In view of (H14), for $\epsilon=\lambda_{0} \omega / 2 e^{L}>0$, there is a constant $\delta>0$ such that

$$
|f(t, s)| \leq \epsilon|s|, \quad \forall 0 \leq|s| \leq \delta, t \in[0, \sigma(T)]_{\mathbb{T}} .
$$

As a result, we have

$$
\left|F\left(\sigma(t), u^{\sigma}(t)\right)\right|=\int_{0}^{u^{\sigma}(t)} f(\sigma(t), s) \mathrm{d} s \leq \int_{0}^{\left|u^{\sigma}(t)\right|} \epsilon|s| \mathrm{d} s=\frac{\epsilon}{2}\left|u^{\sigma}(t)\right|^{2}
$$

which implies from Lemma 3.4 that

$$
\int_{0}^{\sigma(T)}\left|F\left(\sigma(t), u^{\sigma}(t)\right)\right| \Delta t \leq \frac{\epsilon}{2}\left\|u^{\sigma}\right\|_{2}^{2} \leq \frac{\epsilon}{2 \lambda_{0} \omega}\|u\|^{2}, \quad \forall 0 \leq|s| \leq \delta
$$

From the above inequality, (H10) and (H12), it follows that

$$
\varphi(u) \geq \frac{1}{2}\|u\|^{2}-\frac{\epsilon e^{L}}{2 \lambda_{0} \omega}\|u\|^{2}=\frac{1}{4}\|u\|^{2} .
$$


Take $\rho=\delta / a_{0}, \alpha=\delta^{2} / 4 a_{0}^{2}$. By Lemma 3.5, if $\|u\|=\rho$, then $0<\|u\|_{\infty} \leq \delta$. Set $B_{\rho}=\{u \in \mathbb{H} \mid$ $\left.\|u\|<\rho=\delta / a_{0}\right\}$, then $\varphi(u) \geq \alpha>0$ for all $u \in \partial B_{\rho}$. Condition (J1) is satisfied. Therefore, problem (1.1) has at least one weak solution. This completes the proof.

Remark 4.11. Let $C=0, r(t) \equiv 0$ and let $I_{j}(t) \equiv 0$ for all $t \in[0, \sigma(T)]_{\mathbb{T}}, j=1,2, \ldots, p$, then Corollary 4.10 reduces to Theorem 4.2 in [33].

\section{Acknowledgment}

This work is supported by the National Natural Sciences Foundation of People's Republic of China under Grant 10971183.

\section{References}

[1] G. Duffing, Erzwungene Schwingungen bei Veränderlicher Eigenfrequenz und Ihre Technische Beduetung, Sammlung Vieweg, Braunschweig, Germany, 1918.

[2] E. C. Zeeman, "Duffing's equation in brain modelling," Journal of the Institute of Mathematics and Its Applications, vol. 12, no. 7, pp. 207-214, 1976.

[3] F.-G. Xie, W.-M. Zheng, and B.-L. Hao, "Symbolic dynamics of the two-well Duffing equation," Communications in Theoretical Physics, vol. 24, no. 1, pp. 43-52, 1995.

[4] D. Hao and S. Ma, "Semilinear Duffing equations crossing resonance points," Journal of Differential Equations, vol. 133, no. 1, pp. 98-116, 1997.

[5] S. Ma, Z. Wang, and J. Yu, "Coincidence degree and periodic solutions of Duffing equations," Nonlinear Analysis: Theory, Methods E Applications, vol. 34, no. 3, pp. 443-460, 1998.

[6] B. Liu, "Boundedness of solutions for semilinear Duffing equations," Journal of Differential Equations, vol. 145, no. 1, pp. 119-144, 1998.

[7] B. Liu, "Boundedness in nonlinear oscillations at resonance," Journal of Differential Equations, vol. 153, no. 1, pp. 142-174, 1999.

[8] H. Khammari, C. Mira, and J.-P. Carcassés, "Behavior of harmonics generated by a Duffing type equation with a nonlinear damping, part I," International Journal of Bifurcation and Chaos, vol. 15, no. 10, pp. 3181-3221, 2005.

[9] A. Elías-Zúñiga, "A general solution of the Duffing equation," Nonlinear Dynamics, vol. 45, no. 3-4, pp. 227-235, 2006.

[10] S. W. Ma and J. H. Wu, "A small twist theorem and boundedness of solutions for semilinear Duffing equations at resonance," Nonlinear Analysis: Theory, Methods \& Applications, vol. 67, no. 1, pp. 200-237, 2007.

[11] H. Chen and Y. Li, "Rate of decay of stable periodic solutions of Duffing equations," Journal of Differential Equations, vol. 236, no. 2, pp. 493-503, 2007.

[12] A. Caneco, C. Grácio, and J. L. Rocha, "Kneading theory analysis of the Duffing equation," Chaos, Solitons and Fractals, vol. 42, no. 3, pp. 1529-1538, 2009.

[13] M. Bohner and A. Peterson, Dynamic Equations on Time Scales: An Introduction with Applications, Birkhäuser, Boston, Mass, USA, 2001.

[14] V. Spedding, Taming Nature's Numbers, New Scientist, 2003.

[15] M. A. Jones, B. Song, and D. M. Thomas, "Controlling wound healing through debridement," Mathematical and Computer Modelling, vol. 40, no. 9-10, pp. 1057-1064, 2004.

[16] R. P. Agarwal, M. Bohner, and W.-T. Li, Nonoscillation and Oscillation: Theory for Functional Differential Equations, vol. 267 of Monographs and Textbooks in Pure and Applied Mathematics, Marcel Dekker, New York, NY, USA, 2004.

[17] M. Benchohra, J. Henderson, and S. Ntouyas, Impulsive Differential Equations and Inclusions, vol. 2 of Contemporary Mathematics and Its Applications, Hindawi Publishing Corporation, New York, NY, USA, 2006.

[18] W. M. Haddad, V. Chellaboina, and S. G. Nersesov, Impulsive and Hybrid Dynamical Systems, Stability, Dissipativity, and Control, Princeton Series in Applied Mathematics, Princeton University Press, Princeton, NJ, USA, 2006. 
[19] J. J. Nieto and R. Rodríguez-López, "Boundary value problems for a class of impulsive functional equations," Computers $\mathcal{E}$ Mathematics with Applications, vol. 55, no. 12, pp. 2715-2731, 2008.

[20] J. Chu and J. J. Nieto, "Impulsive periodic solutions of first-order singular differential equations," Bulletin of the London Mathematical Society, vol. 40, no. 1, pp. 143-150, 2008.

[21] J. Zhou and Y. Li, "Existence of solutions for a class of second-order Hamiltonian systems with impulsive effects," Nonlinear Analysis: Theory, Methods \& Applications, vol. 72, no. 3-4, pp. 1594-1603, 2010.

[22] Z. Zhang and R. Yuan, "An application of variational methods to Dirichlet boundary value problem with impulses," Nonlinear Analysis: Real World Applications, vol. 11, no. 1, pp. 155-162, 2010.

[23] C. Li, Y. Li, and Y. Ye, "Exponential stability of fuzzy Cohen-Grossberg neural networks with time delays and impulsive effects," Communications in Nonlinear Science and Numerical Simulation, vol. 15, no. 11, pp. 3599-3606, 2010.

[24] Y. Li and T. Zhang, "Existence and uniqueness of anti-periodic solution for a kind of forced Rayleigh equation with state dependent delay and impulses," Communications in Nonlinear Science and Numerical Simulation, vol. 15, no. 12, pp. 4076-4083, 2010.

[25] H. Zhang, W. Xu, and L. Chen, "A impulsive infective transmission SI model for pest control," Mathematical Methods in the Applied Sciences, vol. 30, no. 10, pp. 1169-1184, 2007.

[26] G. Zeng, F. Wang, and J. J. Nieto, "Complexity of a delayed predator-prey model with impulsive harvest and Holling type II functional response," Advances in Complex Systems, vol. 11, no. 1, pp. 7797, 2008.

[27] K. Liu and G. Yang, "Cone-valued-Lyapunov functions and stability for impulsive functional differential equations," Nonlinear Analysis: Theory, Methods E Applications, vol. 69, no. 7, pp. 2184 2191, 2008.

[28] B. Ahmad and J. J. Nieto, "Existence and approximation of solutions for a class of nonlinear impulsive functional differential equations with anti-periodic boundary conditions," Nonlinear Analysis: Theory, Methods E Applications, vol. 69, no. 10, pp. 3291-3298, 2008.

[29] Y. Li, "Positive periodic solutions of nonlinear differential systems with impulses," Nonlinear Analysis: Theory, Methods \& Applications, vol. 68, no. 8, pp. 2389-2405, 2008.

[30] H. Zhang, L. Chen, and J. J. Nieto, "A delayed epidemic model with stage-structure and pulses for pest management strategy," Nonlinear Analysis: Real World Applications, vol. 9, no. 4, pp. 1714-1726, 2008.

[31] Z. Luo and J. J. Nieto, "New results for the periodic boundary value problem for impulsive integrodifferential equations," Nonlinear Analysis: Theory, Methods E Applications, vol. 70, no. 6, pp. 2248-2260, 2009.

[32] Y. Li, X. Chen, and L. Zhao, "Stability and existence of periodic solutions to delayed Cohen-Grossberg BAM neural networks with impulses on time scales," Neurocomputing, vol. 72, no. 7-9, pp. 1621-1630, 2009.

[33] L. Jiang and Z. Zhou, "Existence of weak solutions of two-point boundary value problems for secondorder dynamic equations on time scales," Nonlinear Analysis: Theory, Methods E Applications, vol. 69, no. 4, pp. 1376-1388, 2008.

[34] J. Zhou and Y. Li, "Existence and multiplicity of solutions for some Dirichlet problems with impulsive effects," Nonlinear Analysis: Theory, Methods \& Applications, vol. 71, no. 7-8, pp. 2856-2865, 2009.

[35] M. Bohner and A. Peterson, Advances in Dynamic Equations on Time Scales, Birkhäuser, Boston, Mass, USA, 2003.

[36] B. P. Rynne, " $L^{2}$ spaces and boundary value problems on time-scales," Journal of Mathematical Analysis and Applications, vol. 328, no. 2, pp. 1217-1236, 2007.

[37] R. Agarwal, M. Bohner, and A. Peterson, "Inequalities on time scales: a survey," Mathematical Inequalities $\mathcal{E}$ Applications, vol. 4, no. 4, pp. 535-557, 2001.

[38] J. Mawhin and M. Willem, Critical Point Theory and Hamiltonian Systems, vol. 74 of Applied Mathematical Sciences, Springer, Boston, Mass, USA, 1989.

[39] D. J. Guo, Nonlinear Functional Analysis, Shandong Science and Technology Press, Jinan, China, 2001.

[40] P. H. Rabinowitz, "Minimax methods in critical point theory with applications to differential equations," in Proceedings of the CBMS Regional Conference in the Mathematical Sciences, vol. 65, American Mathematical Society, Providence, RI, USA, 1986.

[41] R. P. Agarwal, M. Bohner, and P. J. Y. Wong, "Sturm-Liouville eigenvalue problems on time scales," Applied Mathematics and Computation, vol. 99, no. 2-3, pp. 153-166, 1999. 


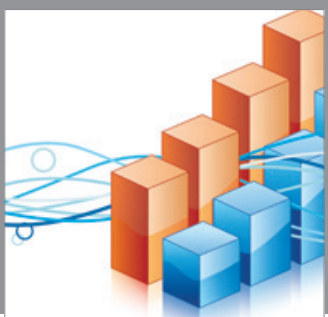

Advances in

Operations Research

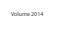

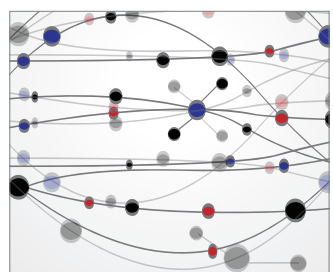

\section{The Scientific} World Journal
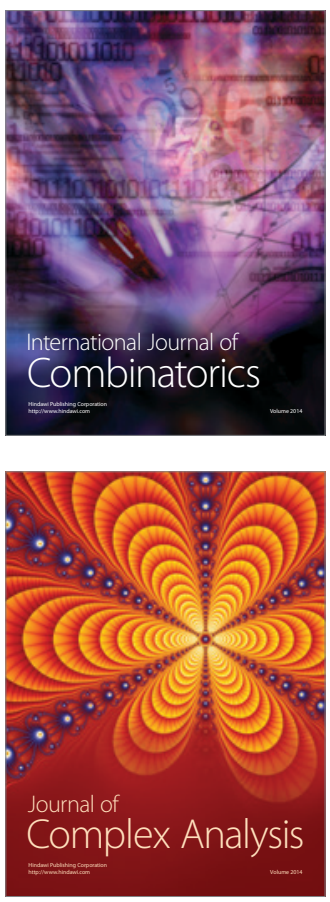

International Journal of

Mathematics and

Mathematical

Sciences
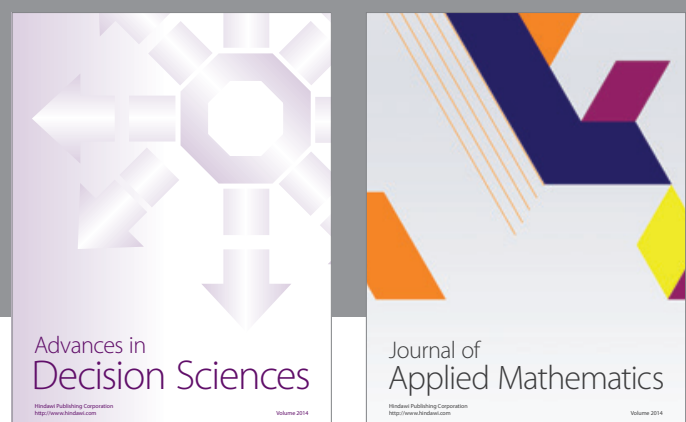

Journal of

Applied Mathematics
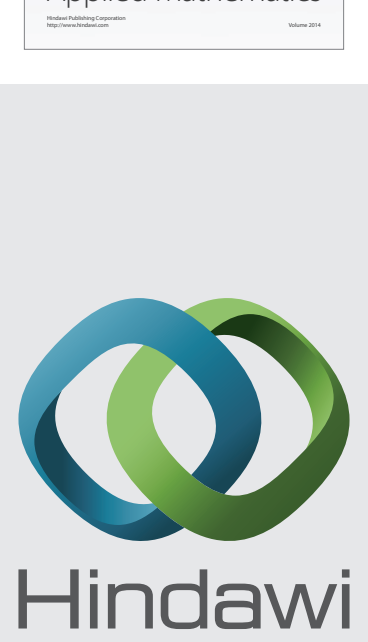

Submit your manuscripts at http://www.hindawi.com
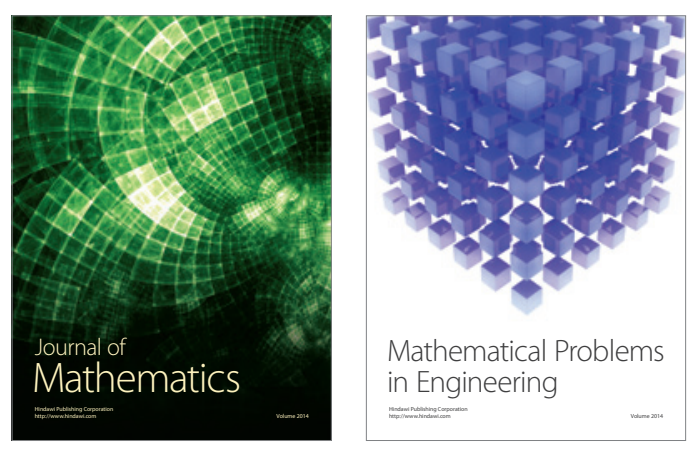

Mathematical Problems in Engineering
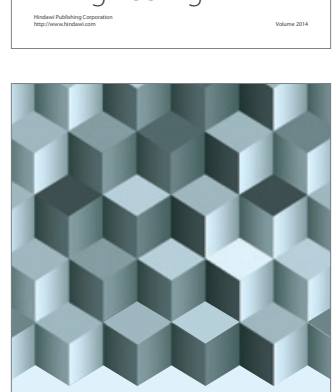

Journal of

Function Spaces
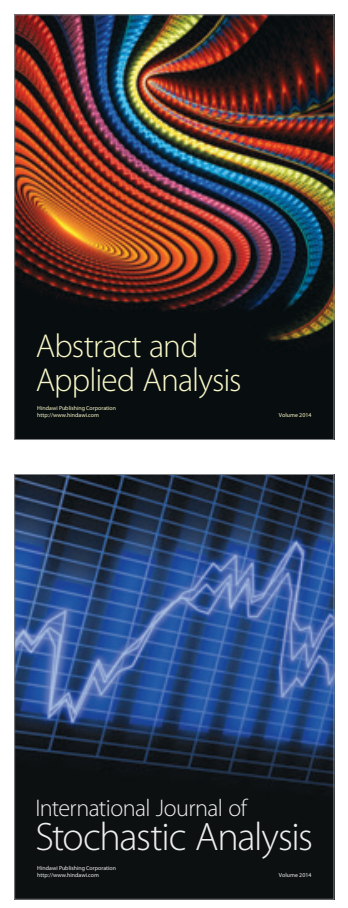

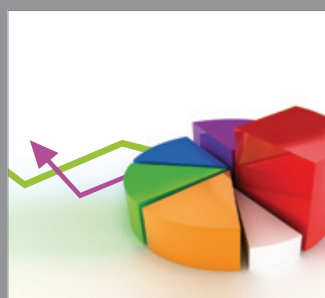

ournal of

Probability and Statistics

Promensencen
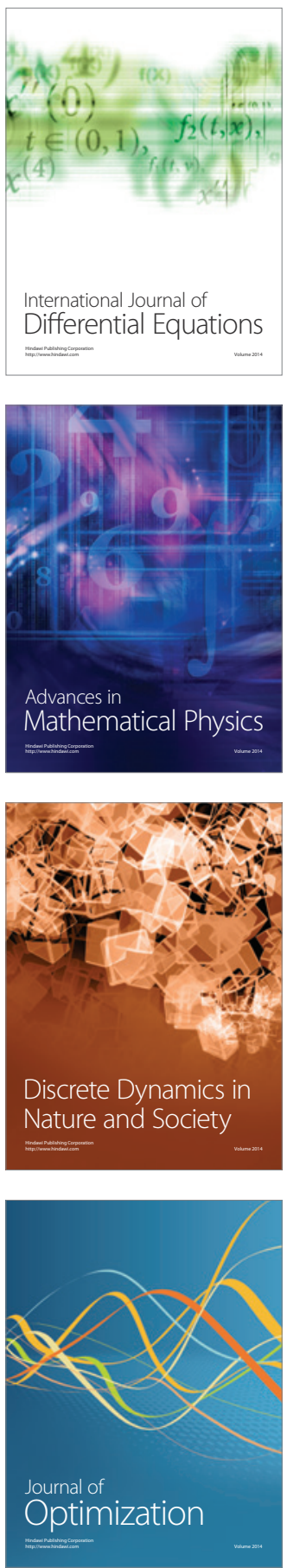